SHORT COMMUNICATION

\title{
Amisulpride vs. fluoxetine treatment of Chronic Fatigue Syndrome: A pilot study
}

\author{
Matteo Pardini a,b,c,*, Silvia Guida ${ }^{a}$, Alberto Primavera ${ }^{a}$, Frank Krueger ${ }^{\text {d,e }}$, \\ Leonardo Cocito $^{a}$, Leonardo Emberti Gialloreti ${ }^{c, f}$
}

\author{
a Department of Neurosciences, Ophthalmology and Genetics, University of Genoa, Genoa, Italy \\ b Magnetic Resonance Research Centre on Nervous System Diseases, University of Genoa, Genoa, Italy \\ c Centre for Communication and Neurorehabilitation Research-CNAPP, Rome, Italy \\ 'Department of Molecular Neuroscience, George Mason University, Fairfax, Virginia, USA \\ e Cognitive Neuroscience Section, National Institute of Neurological Disorders and Stroke-National Institutes of Health, \\ Bethesda, Maryland, USA \\ ${ }^{f}$ Department of Public Health and Cell Biology, Rome Tor Vergata University, Rome, Italy
}

Received 4 July 2010; received in revised form 30 September 2010; accepted 16 October 2010

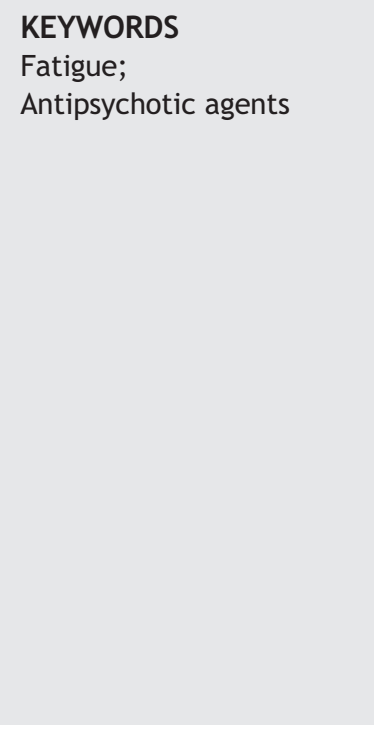

\begin{abstract}
Different pharmacologic agents have been evaluated in the treatment of Chronic Fatigue Syndrome (CFS), albeit with moderate efficacy. Among the compounds thought to present with potential to be efficacious in CFS patients stands out low-dose amisulpride, a substituted benzamide that has been shown to be an useful treatment for conditions which exhibit some overlap with CFS such as dysthymia and somatoform disorders. We thus recruited forty nondepressed CFS patients that were randomized to receive either amisulpride $25 \mathrm{mg}$ bid, or fluoxetine $20 \mathrm{mg}$ uid; all subjects were un-blinded to the treatment regimen. At the time of enrollment in the study and after twelve weeks of treatment, enrolled subjects completed the Krupp Fatigue Severity Scale, the Hospital Anxiety and Depression Scale and a visual analog scale focused on pain and bodily discomfort. Moreover, all subjects were evaluated by a clinician, blinded to the treatment regimen, using the Clinical Global Impression Severity Scale. Our data revealed a significant improvement both in self-report, and observer-based measures for the amisulpride-treated, but not for the fluoxetine-treated patients. Amisulpride-treated subjects also presented with a significant reduction of somatic complaints, while the amisulpride effect on anxiety and mood levels was not significant. Both drugs were equally well tolerated. Summing up, we showed a positive symptomatic effect of amisulpride, compared to SSRI treatment, in a group of non-depressed CSF patients on self-report and on observer-based measures of fatigue
\end{abstract}

\footnotetext{
* Corresponding author. Department of Neurosciences, Ophthalmology and Genetics (DINOG), University of Genoa, Via De Toni, 5 - 16132, Genoa, Italy. Tel.: + 390103537850 ; fax: + 390103538639.

E-mail address: matteo.pardini@gmail.com (M. Pardini).
} 
and somatic complaints. If confirmed by larger, blinded studies, amisulpride thus could represent an effective approach to this difficult-to-treat condition.

(c) 2010 Elsevier B.V. and ECNP. All rights reserved.

\section{Introduction}

Chronic Fatigue Syndrome (CFS) is a disorder with an unclear etiology characterized by disabling physical and mental fatigue, stress-intolerance, widespread pain, and impairment in sleep and concentration, which cannot be better explained by other medical or psychiatric diagnoses (Fukuda et al., 1994).

Different pharmacological agents have been evaluated in CFS patients; to date, triciclycs antidepressants and selective serotonin reuptake inhibitors (SSRI) represent the more widely used drug classes, albeit with mixed results (Pae et al., 2009).

Amisulpride, a substituted benzamide, is an atypical antipsychotic (Green, 2002) that presents potential as an effective treatment in CFS (Green, 2002; Pae et al., 2009). At low doses, amisulpride (100 mg/die or less) preferentially blocks presynaptic dopamine autoreceptors that control dopamine synthesis and release; whereas, at higher doses, it presents postsynaptic dopamine D2-receptor antagonism (Green, 2002). Low-dose amisulpride has been shown to positively impact both negative symptoms in schizophrenia (Green, 2002) and dystimic symtomatology (Rocca et al., 2002). Moreover, substituted benzamides have been successfully used to treat somatoform disorders (Rouillon et al., 2001). As schizophrenia negative symtomatology, somatoform disorders and dystimia, all exhibit some overlap with the clinical presentation of CFS, low-dose amisulpride treatment could be potentially useful in this difficult-totreat population.

Aim of this study was thus to provide preliminary estimates of effect of amisulpride, comparing it to SSRI treatment, on subjects affected by CFS. Primary outcome measure of this efficacy study was symptom alleviation after twelve weeks of treatment, measured by standardized selfreports and pain scales, as well as by clinician's evaluation.

\section{Experimental procedures}

\subsection{Subjects enrollment, treatment and evaluation}

For this study we screened fifty-two subjects with reported CFS; of these fifty-two patients, six subjects were excluded for laboratory tests alterations and other six subjects were excluded on clinical grounds (abnormalities of the neurological examination) thus leading to our experimental cohort of forty subjects (twenty-two females, eighteen males; mean age $31.9 \pm 1.8$ years; mean disease duration $2.5 \pm 1.0$ years). All subjects were enrolled in a single center through referrals from treating clinicians and through self-referrals. The study was not registered in international clinical trials databases, given its preliminary and observational nature, the commercial availability and known safety profile of both drugs, as well as and the patients' lack of blindness for the treatment protocol. Patients included in the study were required to have a diagnosis of CFS according to published criteria (Fukuda et al., 1994). None of the enrolled subjects presented with any neurological or psychiatric comorbiditiy, as assessed by expert clinical evaluation and medical history review. All enrolled subjects reported previous antidepressant use but were free from psychoactive drug use in the six months preceding the enrollment in the study. All enrolled subjects presented with routine laboratory tests within normal ranges including thyroid-stimulating hormone essay.

After enrollment in the study, each subject was randomized to receive either amisulpride $25 \mathrm{mg}$ bid., or fluoxetine $20 \mathrm{mg}$ uid. (active control). Randomization was based on the enrollment list (evennumbered subjects received amisulpride), while subjects' order in the enrollment list was casually generated by an observer blinded to the study design). There was no significant difference in age $(p=0.5)$ or disease duration between the two groups $(p=0.2)$. All the subjects completed the planned 12 -week long therapy period and were aware of which treatment group they were included. At the time of enrollment in the study $\left(t_{0}\right)$ and after twelve weeks of treatment $\left(t_{1}\right)$ all subjects were asked to complete the Krupp Fatigue Severity Scale (FSS) (Krupp et al., 1989) and the Hospital Anxiety and Depression Scale (HADS) (Zigmond and Snaith, 1983).

The FSS is a self-report measure of fatigue, composed of nine items rated from 1 to 7, according to a Likert-type scale, where 1 indicates no fatigue-related impairment and 7 indicates severe impairment. The HADS is a self-report rating scale designed to measure anxiety and depression in subjects with medical conditions through the evaluation of the cognitive but not the somatic aspects of mood and anxiety disorders; it is thus thought to be well-suited to assess CFS patients (Morriss and Wearden, 1998). All subjects presented with HADS scores lower than 7, both for the anxiety (HADS-A) and depression (HADS-D) subscales, thus scoring inside the normal range (Morriss and Wearden, 1998).

In addition, at both time points, subjects completed a visual analog scale aimed to evaluate pain and bodily discomfort subjective intensity (VAS-pain), which scores from 0 (no pain or bodily discomfort) to 100 (maximum pain or bodily discomfort) and a scale of perceived quality of life $(\mathrm{QoL})$ : the Short-Form 12-Item Health Survey (SF-12) (Ware et al., 1996). The SF-12 is a brief selfreport scale of QoL which has been used in different studies of neuropsychiatric conditions (Salyers et al., 2000); higher scores represent higher QoL perception. All subjects, at $t_{1}$, completed also the Frequency, Intensity, and Burden of Side Effects Rating Scale (FIBSER; Wisniewski et al., 2006) to evaluate medications side effects. The FIBSER is a self-report questionnaire that assesses three domains of side effects (frequency, intensity and global burden) using 7-point scales, with higher scores indicating a greater side effect impact.

Lastly, at both study visits, disease burden was evaluated by a clinician blinded to the drug regimen, using the Clinical Global Impression Severity (CGI-S) scale (Guy, 1976). The CGI represent the clinician's interpretation of the severity of a patient's illness and it is rated on a scale from 1 to 7 , with higher scores indicating greater severity.

All subjects gave informed consent to the study. Our institution ethics committee approved all the study procedures.

\subsection{Statistical analysis}

Mann-Whitney $U$ test were used to compare FSS, HADS, VAS-pain, SF-12 and CGI scores at enrollment and at $t_{1}$ and FIBSER scores at $t_{1}$ between the two treatment groups, Wilcoxon matched pairs test was applied to compare differences in FSS, HADS, SF-12, VAS-pain and CGI-S scores evaluated at the two time-points (i.e. to compare scores at enrollment with scores after twelve weeks of treatment) 
within each treatment group. Statistical significance was set at $p$ 0.05 Bonferroni-corrected for multiple comparisons. All data are reported as means \pm standard deviations.

\section{Results}

\subsection{Behavioral evaluation at enrollment}

FSS, VAS-pain, HADS and CGI-S scores at enrolment and after twelve weeks of therapy of the two groups are summarized in Table 1. FSS scores are also plotted in Fig. 1. At the time of enrollment there was no significant difference between the amisulpride and fluoxetine treatment groups in FSS $(50.5 \pm 7.0$ vs. $52.4 \pm 5.7 ; p=0.5)$, VAS-pain $(59.9 \pm 9.5$ vs. $55.9 \pm 9.5$; $\mathrm{p}=0.4$ ), HADS-depression (HADS-D: $4.9 \pm 0.9$ vs. $5.1 \pm 0.8$; $\mathrm{p}=0.5$ ), HADS-anxiety (HADS-A: $5.3 \pm 0.8$ vs. $5.3 \pm 0.9 ; \mathrm{p}=0.7$ ), SF-12 scores $(41.3 \pm 7.0$ vs. $41.7 \pm 7.1 ; p=0.9)$ and $G G I-S$ scores $(4.9 \pm 0.6$ vs. $4.6 \pm 0.9 ; p=0.7)$.

\subsection{Behavioral evaluation after twelve weeks of treatment}

FSS, VAS-pain, HADS and CGI-S scores after twelve weeks of therapy of the two groups are summarized in Table 1. FSS scores are also plotted in Fig. 1. After twelve weeks of treatment the amisulpride-treated subjects, compared to the fluoxetine-treated ones, presented both with significantly lower self-reported fatigue levels (FSS scores: $36.3 \pm 8.6$ vs. $48.9 \pm 4.9 ; p=0.001, z=-4.2$ ) and lower self reported pain and bodily discomfort levels (VAS-pain scores: $40.5 \pm 13.1$ vs. $53.1 \pm 8.3 ; p=0.01, z=-3.1$ ), as well as higher perceived QoL (SF-12 scores: $53.2 \pm 4.8$ vs. $37.6 \pm 4.9$; $\mathrm{p}=0.01, \mathrm{z}=3.6)$ and lower clinician-evaluated disease burden (CGI-S scores: $2.9 \pm 0.6$ vs. $4.2 \pm 1.1 ; p=0.005, z=-2.9$ ). There was no significant difference between the two groups in reported depressive levels (HADS-D scores: $4.1 \pm 0.9$ vs. $4.3 \pm 0.9 ; p=0.6$ ) or anxiety symptoms (HADS-A scores: $4.5 \pm 1.0$ vs. $4.9 \pm 1.0 ; p=0.6$ ).

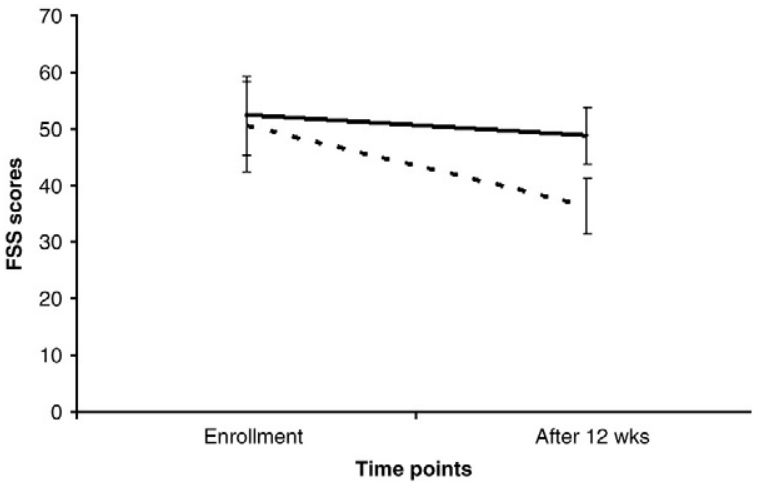

Figure 1 FSS scores at $t_{0}$ and $t_{1}$ for the Amisulpride (dotted line) and Fluoxetine (bold line) groups. Error bars represent standard deviations.

\subsection{Behavioral evaluation differences between $t_{0}$ and $t_{1}$ in the amisulpride group}

There was a significant reduction in FSS $(t=7.2, p=0.001)$, VASpain $(\mathrm{t}=7.8, \mathrm{p}=0.001)$, and $\mathrm{CGI}-\mathrm{S}$ scores $(\mathrm{t}=9.2, \mathrm{p}=0.001)$ between $t_{0}$ and $t_{1}$ in the amisulpride group, while the difference in HADS-A $(t=1.6 ; p=0.14)$ and HADS-D $(t=1.8 ; p=0.1)$ scores between $t_{0}$ and $t_{1}$ did not reach statistical significance. Moreover, there was a significant increase in SF-12 scores $(t=-6.5 ; p=0.001)$ between $t_{0}$ and $t_{1}$ in the amisulpride group.

\subsection{Behavioral evaluation differences between $t_{0}$ and $\mathrm{t}_{1}$ in the fluoxetine group}

There was a significant reduction in HADS-D scores $(t=3.2$, $\mathrm{p}=0.009$ ) between $\mathrm{t}_{0}$ and $\mathrm{t}_{1}$ in the fluoxetine group, while the difference in FSS $(t=1.8 ; p=0.1)$, VAS-pain $(t=1.9 ; p=0.09)$, CGI-S $(t=1.7 ; p=0.1)$, SF-12 $(t=1.6 ; p=0.1)$ and HADS-A $(t=1.8$;

Table 1 Demographical, clinical and neuropsychological data: Amisulpride and Fluoxetine treatment groups.

\begin{tabular}{lcr} 
& Amisulpride group & $\begin{array}{c}\text { Fluoxetine group } \\
\text { (Mean } \pm \text { Std. Deviations) }\end{array}$ \\
\hline Age & (Mean \pm Std.Deviations) & $30.6 \pm 3.2$ \\
Disease Duration & $31.3 \pm 2.2$ & $2.9 \pm 0.9$ \\
FSS at enrolment & $2.5 \pm 1.0$ & $52.4 \pm 5.7$ \\
FSS after 12 wk & $50.5 \pm 7.0$ & $48.9 \pm 4.9$ \\
VAS-pain at enrolment & $36.3 \pm 8.6$ & $55.9 \pm 9.5$ \\
VAS-pain after 12 wk & $59.9 \pm 9.5$ & $53.1 \pm 8.3$ \\
HADS-A at enrolment & $40.5 \pm 13.1$ & $5.3 \pm 0.9$ \\
HADS-A after 12 wk & $5.3 \pm 0.8$ & $4.9 \pm 1.0$ \\
HADS-D at enrolment & $4.5 \pm 1.0$ & $5.1 \pm 0.8$ \\
HADS-D after 12 wk & $4.9 \pm 0.9$ & $4.2 \pm 1.0$ \\
SF-12 at enrolment & $4.3 \pm 0.9$ & $41.7 \pm 7.1$ \\
SF-12 after 12 wk & $41.3 \pm 7.0$ & $37.6 \pm 4.9$ \\
CGI-S at enrolment & $53.2 \pm 4.8$ & $4.6 \pm 0.9$ \\
CGI-S after 12 wk & $4.9 \pm 0.6$ & $4.2 \pm 1.1$ \\
FIBSER-Frequency after 12 wk & $2.9 \pm 0.6$ & $0.8 \pm 0.5$ \\
FIBSER-Intensity after 12 wk & $0.9 \pm 0.7$ & $0.6 \pm 0.4$ \\
FIBSER-Burden after 12 wk & $0.7 \pm 0.5$ & $0.6 \pm 0.8$ \\
\hline
\end{tabular}


$\mathrm{p}=0.8$ ) scores between $t_{0}$ and $t_{1}$ did not reach statistical significance.

\subsection{Side effects perception differences between the amisulpride and fluoxetine groups}

FIBSER scores at $t_{1}$ are reported in Table 1 . There was no difference in FIBSER-Frequency $(z=-0.7 ; p=0.6)$ FIBSER-Intensity $(z=-0.2 ; p=0.9)$ and FIBSER-Burden $(z=-0.6 ; p=0.6)$ between the amisulpride and fluoxetine groups.

\section{Discussion}

Aim of the study was to verify the potential of low-dose amisulpride treatment to ameliorate CFS symtomatology and QoL perception after three months of therapy in a group of non-depressed CFS patients. Our data revealed a significant clinical improvement both in self-report and observer-based measures for the amisulpride-treated subjects but not for the fluoxetine treated patients, while both drugs were equally well tolerated. Amisulpride-treated subjects presented also with a significant reduction of somatic complaints, while the amisulpride effect on anxiety and mood levels was not significant.

Low-dose amisulpride has been shown to bind preferentially extrastriate dopamine receptors (Xiberas et al., 2001) and, in schizophrenic patients, to increase frontal blood flow and to partially improve frontal cognitive functions (Vaiva et al., 2002). Interestingly, frontal lobes seem to play a significant role in fatigue perception, as prefrontal structural alterations have been related with fatigue in different conditions, such as penetrating brain injury, (Pardini et al., 2010). Additionally, successful cognitive-behavioral CFS treatment has been linked with frontal grey matter modulation (de Lange et al., 2008). The relationship between amisulpride and frontal lobe activity and the link between frontal lobe functions and fatigue seem to suggest that the observed effect of amisulpride on fatigue levels could be mediated trough prefrontal cortex activity modulation.

Furthermore, recent studies point to a 5-HT7 receptor antagonist activity in addition to its effects on dopaminergic receptors as a possible substrate for amisulpride action in mood disorders (Abbas et al., 2009). 5-HT7 receptors are thought to play a role in memory and sleep (Hedlund and Sutcliffe, 2004); as sleep architecture alterations and memory complaints are often found in CFS patients, amisulpride activity at 5-HT7 receptor level could also positively affect sleep quality and cognition in CSF patients, thus contributing to its efficacy in these patients.

In conclusion, in a group of CSF patients, we observed a positive effect of amisulpride, compared to SSRI treatment, on self-report and observer-based measures of fatigue, QoL and disease burden. Amisulpride treatment was well tolerated. The main limitations of this pilot study are its observational, un-blinded design, and the moderate number of enrolled subjects. Larger, registered randomized clinical trials are warranted to confirm our observation and to explore the neurobiological basis of amisulpride effects in CFS.

\section{Role of the Funding Source}

None.

\section{Contributors}

Authors Pardini and Emberti Gialloreti designed the study and wrote the protocol. Authors Pardini, Guida and Emberti Gialloreti recruited the patients. Authors Guida, Primavera, Krueger, Cocito and Emberti Gialloreti managed the literature searches and the statistical analyses. Authors Pardini and Emberti wrote first draft of the manuscript. All authors contributed to and have approved the final manuscript.

All individuals included as authors have contributed substantially to the scientific process leading up to the writing of the paper. All coauthors have seen and agree with the contents of the submitted manuscript which is not under consideration elsewhere.

\section{Conflict of Interest}

None to report.

\section{References}

Abbas, A.I., Hedlund, P.B., Huang, X.P., Tran, T.B., Meltzer, H.Y., Roth, B.L., 2009. Amisulpride is a potent 5-HT7 antagonist: relevance for antidepressant actions in vivo. Psychopharmacology 205 (1), 119-128 (Berl).

de Lange, F.P., Koers, A., Kalkman, J.S., Bleijenberg, G., Hagoort, P., van der Meer, J.W., Toni, I., 2008. Increase in prefrontal cortical volume following cognitive behavioural therapy in patients with chronic fatigue syndrome. Brain 131 (Pt 8), 2172-2180

Fukuda, K., Straus, S.E., Hickie, I., Sharpe, M.C., Dobbins, J.G., Komaroff, A., 1994. The chronic fatigue syndrome: a comprehensive approach to its definition and study. International Chronic Fatigue Syndrome Study Group. Ann. Intern. Med. 121 (12), 953-959.

Green, B., 2002. Focus on amisulpride. Curr. Med. Res. Opin. 18 (3), 113-117.

Guy, W. (Ed.), 1976. ECDEU Assessment Manual for Psychopharmacology. US Department of Health, Education, and Welfare Public Health Service Alcohol, Drug Abuse, and Mental Health Administration, Rockville, MD.

Hedlund, P.B., Sutcliffe, J.G., 2004. Functional, molecular and pharmacological advances in 5-HT7 receptor research. Trends Pharmacol. Sci. 25 (9), 481-486.

Krupp, L.B., LaRocca, N.G., Muir-Nash, J., Steinberg, A.D., 1989. The fatigue severity scale. Application to patients with multiple sclerosis and systemic lupus erythematosus. Arch. Neurol. 46 (10), 1121-1123.

Morriss, R.K., Wearden, A.J., 1998. Screening instruments for psychiatric morbidity in chronic fatigue syndrome. J. R. Soc. Med. 91 (7), 365-368.

Pae, C.U., Marks, D.M., Patkar, A.A., Masand, P.S., Luyten, P., Serretti, A., 2009. Pharmacological treatment of chronic fatigue syndrome: focusing on the role of antidepressants. Expert Opin. Pharmacother. 10 (10), 1561-1570.

Pardini, M., Krueger, F., Raymont, V., Grafman, J., 2010. Ventromedial prefrontal cortex modulates fatigue after penetrating traumatic brain injury. Neurology 74 (9), 749-754.

Rocca, P., Fonzo, V., Ravizza, L., Rocca, G., Scotta, M., Zanalda, E., Bogetto, F., 2002. A comparison of paroxetine and amisulpride in 
the treatment of dysthymic disorder. J. Affect. Disord. 70 (3), 313-317.

Rouillon, F., Rahola, G., Van Moffaert, M., Lopes, R.G., Dunia, I., 2001. Sulpiride in the treatment of somatoform disorders: results of a European observational study to characterize the responder profile. J. Int. Med. Res. 29 (4), 304-313.

Salyers, M.P., Bosworth, H.B., Swanson, J.W., Lamb-Pagone, J., Osher, F.C., 2000. Reliability and validity of the SF-12 health survey among people with severe mental illness. Med. Care 38, 1141-1150.

Vaiva, G., Thomas, P., Llorca, P.M., Dupont, S., Cottencin, O., Devos, P., Mazas, O., Rascle, C., Steinling, M., Goudemand, M., 2002. SPECT imaging, clinical features, and cognition before and after low doses of amisulpride in schizophrenic patients with the deficit syndrome. Psychiatry Res. 115 (1-2), 37-48.
Ware Jr., J.E., Kosinski, M., Keller, S.D., 1996. A 12 Item Short Form Health Survey: construction of scales and preliminary tests of reliability and validity. Med. Care 34, 220-233.

Wisniewski, S.R., Rush, A.J., Balasubramani, G.K., Trivedi, M.H., Nierenberg, A.A., 2006. Self-rated global measure of the frequency, intensity, and burden of side effects. J. Psychiatr. Pract. 12 (2), 71-79.

Xiberas, X., Martinot, J.L., Mallet, L., Artiges, E., Canal, M., Loc'h, C., Maziere, B., Paillere-Martinot, M.L., 2001. In vivo extrastriatal and striatal D2 dopamine receptor blockade by amisulpride in schizophrenia. J. Clin. Psychopharmacol. 21 (2), 207-214.

Zigmond, A.S., Snaith, R.P., 1983. The hospital anxiety and depression scale. Acta Psychiatr. Scand. 67 (6), 361-370. 